\title{
Impact of structural changes on dielectric and thermal properties of vinylidene fluoride-trifluoroethylene-based terpolymer/copolymer blends
}

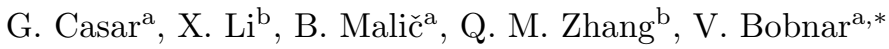 \\ ${ }^{a}$ Jožef Stefan Institute and Jožef Stefan International Postgraduate School, Jamova 39, SI-1000 Ljubljana, Slovenia \\ ${ }^{b}$ Department of Electrical Engineering and Materials Research Institute, The Pennsylvania State University, University \\ Park, Pennsylvania 16802, USA
}

\begin{abstract}
We report dielectric and thermal properties of the poly(vinylidene fluoride-trifluoroethylene-chlorofluoroethylene) terpolymer $[\mathrm{P}(\mathrm{VDF}-\mathrm{TrFE}-\mathrm{CFE})$, a member of the relaxor polymer family that exhibits fast response speeds, giant electrostriction, high electric energy density, and large electrocaloric effect] blended with the ferroelectric poly(vinylidene fluoride-trifluoroethylene) copolymer, $\mathrm{P}(\mathrm{VDF}-\mathrm{TrFE})$. Although the differential scanning calorimetry (DSC) clearly reveal that both components form separate crystalline phases, at low copolymer content blends entirely exhibit a relaxorlike linear dielectric response, since the interfacial couplings to the bulky defects in the terpolymer convert the normal ferroelectric copolymer into a relaxor. On the other hand, dielectric experiments evidence that in blends with 20-50 wt. \% of P(VDF-TrFE) the ferroelectric and relaxor states coexist. This coexistence is confirmed by DSC results, which further reveal the influence of blending on the terpolymer crystallinity and melting point. At last, the crystallinity data appropriately explain the variation of the dielectric constant in $\mathrm{P}(\mathrm{VDF}-\mathrm{TrFE}-\mathrm{CFE}) / \mathrm{P}(\mathrm{VDF}-\mathrm{TrFE})$ blends.
\end{abstract} Keywords: relaxor, ferroelectric, polymer blends, dielectric spectroscopy, DSC

\section{Introduction}

Electroactive polymers based on polyvinylidene fluoride, PVDF, and its copolymer with trifluoroethylene, $\mathrm{P}(\mathrm{VDF}-\mathrm{TrFE})$, are of great interest for a broad range of applications, as they exhibit fast response speeds, and strain levels far above those of traditional piezoceramic materials $[1,2,3]$. For example, a giant electrostriction in electron-irradiated $\mathrm{P}(\mathrm{VDF}-\mathrm{TrFE})$ copolymer [4] and in terpolymers with chlorotrifluoroethylene, $\mathrm{P}(\mathrm{VDF}-\mathrm{TrFE}-\mathrm{CTFE})$ [5, 6], or chlorofluoroethylene, $\mathrm{P}(\mathrm{VDF}-\mathrm{TrFE}-\mathrm{CFE}$ ) [7], has been detected. These systems exhibit a typical relaxor behavior [8], suggesting that electron-irradiation or introduction of

\footnotetext{
*Corresponding author. Tel.: +38614773172, Fax: +38612519385

Email address: vid.bobnar@ijs.si (V. Bobnar) 
CTFE/CFE monomers converts the coherent polarization domain, i.e., all-trans chains, in normal ferroelectric $\mathrm{P}(\mathrm{VDF}-\mathrm{TrFE})$ into nanopolar regions, thus transforming the material into a relaxor system (a relaxor state is usually described as a network of randomly interacting polar nanoregions which are embedded in a highly polarizable medium) $[9,10]$. Concomitantly, an ultrahigh strain response in relaxor polymers is generated due to expansion of the polar regions under an external electric field, coupled with a large difference in the lattice strain between polar and nonpolar phases [4].

Ferroelectric and relaxor polymers have in recent years also been suggested for various advanced applications, as they possess high electric energy density with fast discharge speed [11] and large electrocaloric effect near room temperature [12]. Most of the investigations have, however, focused on either normal ferroelectric polymers or polymers that are completely transformed into a relaxor (e.g., terpolymers or $\mathrm{P}(\mathrm{VDF}-\mathrm{TrFE})$ copolymer, irradiated with a high dose). Only recently properties of $\mathrm{P}(\mathrm{VDF}-\mathrm{TrFE})$ copolymer, irradiated with low and moderate doses of high-energy electrons, have been reported - a clear evidence that ferroelectric and relaxor states coexist in the system has been provided on the basis of dielectric and thermal investigations and it has furthermore been shown that such a coexistence strongly influences some materials' properties [13]. Since irradiation also creates some undesirable side effects (crosslinking of polymer chains or formation of radicals, for example), we have developed a polymer system, where similar coexistence of states could be expected - blends of a relaxor terpolymer and ferroelectric copolymer.

We report dielectric and thermal properties of $\mathrm{P}(\mathrm{VDF}-\mathrm{TrFE}-\mathrm{CFE})$ terpolymer/P(VDF-TrFE) copolymer blends. We show that blends entirely exhibit a relaxorlike linear dielectric response at low copolymer content, while in samples with 20-50 wt. \% of $\mathrm{P}(\mathrm{VDF}-\mathrm{TrFE})$ the ferroelectric and relaxor states coexist, in terms of the fact that samples are comprised of the phase separated relaxor terpolymer and ferroelectric copolymer and that their overall response evolves from relaxorlike towards the ferroelectric on increasing the $\mathrm{P}(\mathrm{VDF}-\mathrm{TrFE})$ content. This coexistence is confirmed by the differential scanning calorimetry, which further reveals the influence of blending on polymers crystallinity and melting points. Moreover, the variation of the dielectric constant in $\mathrm{P}(\mathrm{VDF}-\mathrm{TrFE}-\mathrm{CFE}) / \mathrm{P}(\mathrm{VDF}-\mathrm{TrFE})$ blends is explained in terms of the crystallinity data. A polymer blend approach is thus presented as a promising method for tailoring the dielectric response of a relaxor polymer system.

\section{Materials and methods}

$\mathrm{P}(\mathrm{VDF}-\mathrm{TrFE}-\mathrm{CFE})$ terpolymer and $\mathrm{P}(\mathrm{VDF}-\mathrm{TrFE})$ copolymer powders $(62.5 / 29 / 8.5$ and 55/45 mol. \%, respectively), synthesized by a suspension polymerization method, were dissolved in N,N-dimethylformamide 
at room temperature. Then, the two solutions were mixed together by proper ratios for different blend compositions. The final solution was filtered using $0.2 \mu \mathrm{m}$ sized polytetrafluoroethylene filters and then cast on cleaned glass plates and dried at $70{ }^{\circ} \mathrm{C}$ for $24 \mathrm{~h}$. Afterwards, the films were peeled off from the glass plates and further annealed at $70{ }^{\circ} \mathrm{C}$ for $24 \mathrm{~h}$.

For dielectric measurements, surfaces of 11-15 $\mu \mathrm{m}$ thick polymer films were covered by sputtered electrodes (100 nm of gold on $10 \mathrm{~nm}$ of chromium for better adhesion, diameter of 4-6 mm). Complex dielectric constant $\varepsilon^{*}(\omega, T)=\varepsilon^{\prime}-i \varepsilon^{\prime \prime}$ was measured in the frequency range of $30 \mathrm{~Hz}-1 \mathrm{MHz}$ by using $\mathrm{HP} 4284 \mathrm{~A}$ Precision LCR Meter. The amplitude of the probing ac electric signal was $0.1 \mathrm{~V}$. After heating the samples up to $375 \mathrm{~K}$, the dielectric response was detected during cooling runs with the rate of $0.5 \mathrm{~K} / \mathrm{min}$. The differential scanning calorimetry (DSC) curves were recorded on a Netzsch DSC 204 F1 calorimeter. The sample was packed in an $\mathrm{Al}$ crucible with a lid, loaded into the calorimeter and heated up to $473 \mathrm{~K}$ with a heating rate of $10 \mathrm{~K} / \mathrm{min}$. The temperatures of the phase transitions are reported as the maxima of the peaks. The enthalpy change at the melting point was calculated from the area of the peak, while the temperature and enthalpy calibrations were performed prior to the measurements using standard calibration materials with well-defined transition temperatures and enthalpies.

\section{Results and discussion}

Figure 1 shows the temperature dependences of the real, $\varepsilon^{\prime}$, and imaginary, $\varepsilon^{\prime \prime}$, parts of the complex dielectric constant, detected at various frequencies in the pure $\mathrm{P}(\mathrm{VDF}-\mathrm{TrFE}-\mathrm{CFE})$ terpolymer (100:0), its blends with different wt. \% (5-50) of $\mathrm{P}(\mathrm{VDF}-\mathrm{TrFE})$ copolymer, and in the pure copolymer (0:100). The terpolymer exhibits a typical relaxor broad dispersive maximum, i.e., temperatures at which $\varepsilon^{\prime}$ and $\varepsilon^{\prime \prime}$ exhibit maximum are dependent on frequency. Similar as in inorganic relaxors (where the relaxor behavior is usually correlated with the cationic disorder in the same crystallographic site), this peak is a consequence of polar nanoregions' dynamics and does not denote any phase transition, just as being the case in dipolar glasses $[8,14]$. A small admixture of the $\mathrm{P}(\mathrm{VDF}-\mathrm{TrFE})$ copolymer does not qualitatively influence this spectra, but only slightly increases the maximum values. However, in samples with higher copolymer amount $(\geq 20$ wt. \%) a frequency-independent peak due to the ferroelectric phase transition in the copolymer starts to form in addition to the broad terpolymer's relaxor maximum, which clearly indicates that in these blends ferroelectric and relaxor states coexist. The dielectric spectra of the pure copolymer distinctively shows this ferroelectric phase transition, and, in addition, a dielectric relaxation at lower temperatures. It should therefore be stressed here that in polymer systems the ferroelectric phase transition and/or relaxor 
dynamic behavior take place only in the crystalline part of the system. It is namely well known, from x-ray, heat capacity, and infrared investigations, that PVDF and its copolymers are semicrystalline systems comprising noncrystalline, i.e., amorphous regions, and crystalline regions with a spontaneous polarization associated with parallel packing of all-trans polymer chains $[2,15]$. While on heating the crystalline region adopted ferroelectric or relaxor phase undergoes a transition into a paraelectric phase, and finally melts, the amorphous region undergoes a transition from glassy to a rubbery state below room temperature [15]. This glass transition (sometimes called $\beta$-process) [16] is dynamically manifested as an additional dielectric relaxation in the temperature region of $250-300 \mathrm{~K}[16,17]$ and is in fact present in all our investigated samples - it is only much less pronounced in samples which comprise terpolymer, since it is overrided by the broad relaxor maximum.

The dielectric response thus reveals that ferroelectric and relaxor states coexist in the $\mathrm{P}(\mathrm{VDF}-\mathrm{TrFE}-$ $\mathrm{CFE})$ terpolymer/P(VDF-TrFE) copolymer blends with 20-50 wt. \% of $\mathrm{P}(\mathrm{VDF}-\mathrm{TrFE})$. This conclusion is confirmed by the DSC data, shown in Fig. 2 - besides the melting peaks, which correspond to the melting of the terpolymer around $400 \mathrm{~K}$ and that of the copolymer around $430 \mathrm{~K}$, also the ferroelectric phase transition peak can clearly be seen not only in the pure copolymer sample, but also in 50:50 and 60:40 samples. DSC data, moreover, via two independent melting peaks clearly reveal that both components form separate crystalline phases, indicating that all our blend polymer samples are in fact comprised of the phase separated $\mathrm{P}(\mathrm{VDF}-\mathrm{TrFE}-\mathrm{CFE})$ terpolymer and $\mathrm{P}(\mathrm{VDF}-\mathrm{TrFE})$ copolymer. While the latter exhibits the ferroelectric character for its content of 20-50 wt. \%, no sign of ferroelectricity has been detected in samples with low ( $\leq 15$ wt. \%) copolymer amount. Furthermore, the x-ray diffraction data indicate that the basic crystallographic structure in 95:5 and 90:10 samples remains the same as that of the terpolymer [18]. It thus seems that at a low copolymer content the interfacial couplings to the bulky defects in the terpolymer convert the normal ferroelectric copolymer into a relaxor and, consequently, these blends entirely exhibit a relaxorlike dielectric response.

DSC results are summarized in Table 1. As a consequence of the fact that the melting temperature is reduced with decreased size of crystallites [19] the melting peak temperature of each component, $T_{\text {ter }}$ and $T_{\mathrm{cop}}$, is decreasing in blends with its decreasing weight ratio. This is more clearly revealed for the copolymer part, since its weight ratio covers a wide range of $100 \mathrm{wt}$ \% down to $5 \mathrm{wt}$ \% in our samples. The decreasing of the corresponding melting peak temperature from $429.3 \mathrm{~K}$ in the pure copolymer down to $421.0 \mathrm{~K}$ in the 95:5 blend sample can thus nicely be followed in Fig. 2 .

From the areas of the peaks also the total enthalpy change of the melting $\left(\Delta H_{\text {ter }}, \Delta H_{\text {cop }}\right)$ and the 
normalized values (the actual heat of melting divided by the weight ratio, $\Delta H_{\mathrm{n}, \mathrm{ter}}, \Delta H_{\mathrm{n}, \mathrm{cop}}$ ) have been determined. Then, the relative crystallinity of each component has been deduced by assuming that the enthalpy of the melting is directly proportional to the crystallinity in the sample [16] - the normalized enthalpy change of the melting peak detected in a blend system has thus been divided by the value obtained in the pure terpolymer or copolymer sample, yielding the relative crystallinity values $C_{t e r}=\Delta H_{\mathrm{n}, \operatorname{ter}} / \Delta H_{\mathrm{n}, \operatorname{ter}(100: 0)}$ and $C_{c o p}=\Delta H_{\mathrm{n}, \mathrm{cop}} / \Delta H_{\mathrm{n}, \mathrm{cop}(0: 100)}$ of the terpolymer and copolymer part, respectively.

The most interesting finding here is the fact that at a low copolymer content $(\leq 15 \mathrm{wt}$. \%) the crystallinity of the terpolymer component is increased. It seems that some thin copolymer lamellae serve as nucleation center and favor the crystallization process of the terpolymer. Consequently, due to larger electrical polarization which mostly originates in the crystalline phase, the dielectric constant of these blends is higher than that of the pure terpolymer. If we compare maximum $\varepsilon^{\prime}$ values of 100:0, 95:5, 90:10, and 85:15 blends in Fig. 1 we see that they directly scale with the relative crystallinity data - for example, $\varepsilon_{\max }^{\prime}=53$ in 95:5 sample is 1.15 times higher from the value of $\varepsilon_{\max }^{\prime}=46$ in a pure terpolymer, which is almost exactly the ratio of the crystallinities obtained in these two samples. Therefore, besides tuning the electrocaloric effect [18], a polymer blend approach seems a promising method for tailoring the dielectric response of a relaxor polymer system.

\section{Summary}

Dielectric and thermal properties of relaxor $\mathrm{P}(\mathrm{VDF}-\mathrm{TrFE}-\mathrm{CFE})$ terpolymer blended with the ferroelectric $\mathrm{P}(\mathrm{VDF}-\mathrm{TrFE})$ copolymer are reported. The terpolymer itself exhibits a typical relaxor broad dispersive dielectric maximum, and although both components form separate crystalline phases, at low copolymer content blends entirely exhibit a relaxorlike dielectric response, since the interfacial couplings to the bulky defects in the terpolymer convert the normal ferroelectric copolymer into a relaxor. A small admixture of $\mathrm{P}(\mathrm{VDF}-\mathrm{TrFE})$ thus doesn't qualitatively influence the spectra, but only slightly increases the maximum values of the dielectric constant.

On the other hand, in blends with $20-50$ wt. $\%$ of $\mathrm{P}(\mathrm{VDF}-\mathrm{TrFE})$ the ferroelectric and relaxor states coexist. In the dielectric spectra of these samples a frequency-independent peak due to the ferroelectric phase transition in the copolymer starts to form in addition to the broad terpolymer's relaxor maximum. This coexistence is confirmed by the differential scanning calorimetry, which, via two independent melting peaks, reveal that both components form separate crystalline phases, indicating that all our blend polymer samples are in fact comprised of the phase separated $\mathrm{P}(\mathrm{VDF}-\mathrm{TrFE}-\mathrm{CFE})$ terpolymer and $\mathrm{P}(\mathrm{VDF}-\mathrm{TrFE})$ copolymer. 
DSC data further reveal the influence of blending on the crystallinity and melting points of both polymer components. It is thus shown that, as a consequence of the fact that the melting temperature is reduced with decreased size of crystallites, the melting peak temperature of each component is decreasing in blends with its decreasing weight ratio - for the copolymer part it decreases from $429.3 \mathrm{~K}$ in the pure $\mathrm{P}(\mathrm{VDF}-\mathrm{TrFE})$ down to $421.0 \mathrm{~K}$ in the $95: 5$ blend sample. Finally, the relative crystallinities have been obtained from the normalized entalphy changes at melting, revealing that at a low copolymer content the crystallinity of the terpolymer component is increased. This result explains that the increasing of the dielectric constant in the 95:5, 90:10, and 85:15 blends in comparison to the pure terpolymer is due to larger electrical polarization, which is known to mostly originate in the crystalline phase of a polymer system.

Blending a relaxor terpolymer with the ferroelectric copolymer thus resulted in the system with coexisting ferroelectric and relaxor states, showing similar properties to $\mathrm{P}(\mathrm{VDF}-\mathrm{TrFE})$ copolymer, irradiated with low and moderate doses of high-energy electrons, where such a coexistence has already been shown to strongly influence some materials' properties [13]. Moreover, in the same way as the ferroelectric $\mathrm{P}(\mathrm{VDF}-\mathrm{TrFE})$ evolves, via intermediate states, towards a relaxor system on increasing the dose of high-energy electrons irradiation, here the overall response of $\mathrm{P}(\mathrm{VDF}-\mathrm{TrFE}-\mathrm{CFE})$ terpolymer/P(VDF-TrFE) copolymer blends evolves from relaxor towards the ferroelectric on increasing the $\mathrm{P}(\mathrm{VDF}-\mathrm{TrFE})$ content. Our polymer blend approach could thus be suggested as a promising method for tailoring various properties of a relaxor polymer system, not only the dielectric and thermal response, being reported in this work.

\section{Acknowledgements}

This research was supported by the Slovenian Research Agency under program P1-0125 and by the U.S. DoE, Office of Basic Energy Sciences, Division of Materials Science and Engineering under Award No. DE-FG02-07ER46410.

\section{References}

[1] H. Kawai, Jpn. J. Appl. Phys. 8 (1969) 975.

[2] A. J. Lovinger, Science 220 (1983) 1115.

[3] K. Tashiro, In: Ferroelectric Polymers, H. Nalwa (Ed.), Marcel Dekker, New York, 1995, pp. 63.

[4] Q. M. Zhang, V. Bharti, and X. Zhao, Science 280 (1998) 2101.

[5] H. Xu, Z.-Y. Cheng, D. Olson, T. Mai, and Q. M. Zhang, Appl. Phys. Lett. 78 (2001) 2360.

[6] G. S. Buckley, C. M. Roland, R. Casalini, A. Petchsuk, and T. C. Chung, Chem. Mater. 14 (2002) 2590.

[7] F. Xia, Z.-Y. Cheng, H. Xu, H. Li, Q. M. Zhang, G. J. Kavarnos, R. Y. Ting, G. Abdul-Sedat, and K. D. Belfield, Adv. Mater. 14 (2002) 1574. 
[8] V. Bobnar, B. Vodopivec, A. Levstik, M. Kosec, B. Hilczer, and Q. M. Zhang, Macromolecules 36 (2003) 4436.

[9] D. Viehland, S. J. Jang, L. E. Cross, and M. Wuttig, Phys. Rev. B 46 (1992) 8003.

[10] V. Bobnar, Z. Kutnjak, R. Pirc, and A. Levstik, Phys. Rev. B 60 (1999) 6420.

[11] B. Chu, X. Zhou, K. Ren, B. Neese, M. Lin, Q. Wang, F. Bauer, and Q. M. Zhang, Science 313 (2006) 334.

[12] B. Neese, B. Chu, S.-G. Lu, Y. Wang, E. Furman, and Q. M. Zhang, Science 321 (2008) 821.

[13] G. Casar, X. Li, J. Koruza, Q. M. Zhang, and V. Bobnar, J. Mater. Sci. 48 (2013) 7920.

[14] A. Levstik, Z. Kutnjak, C. Filipič, and R. Pirc, Phys. Rev. B 57 (1998) 11204.

[15] T. Furukawa, Y. Tajitsu, X. Zhang, and G. E. Johnson, Ferroelectrics 135 (1992) 401.

[16] V. Bharti, H. S. Xu, G. Shanthi, and Q. M. Zhang, J. Appl. Phys. 87 (2000) 452.

[17] T. Furukawa, Phase Transit. 18 (1989) 143.

[18] X.-Z. Chen, X.-S. Qian, X. Li, S. G. Lu, H.-M. Gu, M. Lin, Q.-D. Shen, and Q. M. Zhang, Appl. Phys. Lett. 100 (2012) 222902.

[19] Z.-Y. Cheng, D. Olson, H. S. Xu, F. Xia, J. S. Hundal, Q. M. Zhang, F. B. Bateman, G. J. Kavarnos, and T. Ramotowski, Macromolecules 35 (2002) 664 . 
Table 1: DSC melting peak temperatures $\left(T_{\mathrm{ter}}, T_{\mathrm{cop}}\right)$, total $\left(\Delta H_{\mathrm{ter}}, \Delta H_{\mathrm{cop}}\right)$ and normalized $\left(\Delta H_{\mathrm{n}, \mathrm{ter}}, \Delta H_{\mathrm{n}, \mathrm{cop}}\right)$ enthalpy change of the melting peaks, and the relative crystallinities $\left(C_{t e r}=\Delta H_{\mathrm{n}, \operatorname{ter}} / \Delta H_{\mathrm{n}, \operatorname{ter}(100: 0)}, C_{c o p}=\Delta H_{\mathrm{n}, \operatorname{cop}} / \Delta H_{\mathrm{n}, \operatorname{cop}(0: 100)}\right)$ of the terpolymer and copolymer components in the $\mathrm{P}(\mathrm{VDF}-\mathrm{TrFE}-\mathrm{CFE}) / \mathrm{P}(\mathrm{VDF}-\mathrm{TrFE})$ blends.

\begin{tabular}{ccccccccc} 
blend & $T_{\text {ter }}(\mathrm{K})$ & $\Delta H_{\text {ter }}(\mathrm{J} / \mathrm{g})$ & $T_{\text {cop }}(\mathrm{K})$ & $\Delta H_{\text {cop }}(\mathrm{J} / \mathrm{g})$ & $\Delta H_{\mathrm{n}, \text { ter }}(\mathrm{J} / \mathrm{g})$ & $\Delta H_{\mathrm{n}, \mathrm{cop}}(\mathrm{J} / \mathrm{g})$ & $C_{\text {ter }}$ & $C_{\text {cop }}$ \\
\hline $100: 0$ & 400.6 & -14.34 & & & -14.34 & & 1 & \\
$95: 5$ & 398.0 & -15.78 & 421.0 & -0.34 & -16.61 & -6.80 & 1.16 & 0.23 \\
$90: 10$ & 399.6 & -13.27 & 422.5 & -0.88 & -14.74 & -8.76 & 1.03 & 0.30 \\
$85: 15$ & 399.0 & -13.03 & 423.0 & -1.71 & -15.33 & -11.34 & 1.07 & 0.39 \\
$80: 20$ & 399.8 & -10.07 & 426.2 & -3.20 & -12.59 & -16.03 & 0.88 & 0.55 \\
$70: 30$ & 399.7 & -6.05 & 426.5 & -5.31 & -8.64 & -17.7 & 0.60 & 0.60 \\
$60: 40$ & 399.7 & -4.71 & 426.5 & -8.34 & -7.45 & -20.84 & 0.52 & 0.71 \\
$50: 50$ & 398.6 & -2.31 & 426.7 & -11.39 & -4.60 & -22.78 & 0.32 & 0.78 \\
$0: 100$ & & & 429.3 & -29.31 & & -29.31 & & 1 \\
\hline
\end{tabular}



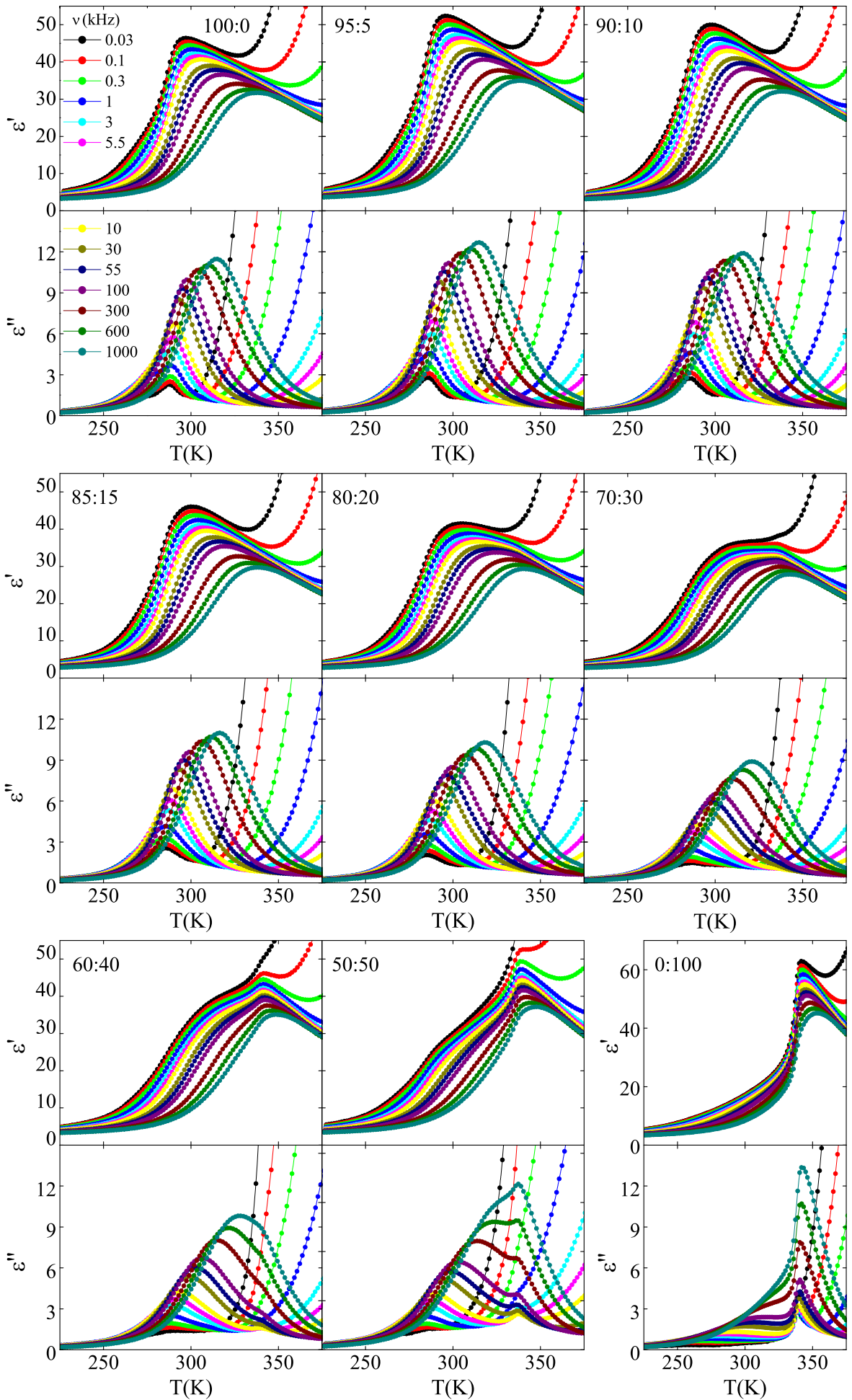

Figure 1: Temperature dependences of the real, $\varepsilon^{\prime}$, and imaginary, $\varepsilon^{\prime \prime}$, parts of the complex dielectric constant, detected at various frequencies in the pure $\mathrm{P}(\mathrm{VDF}-\mathrm{TrFE}-\mathrm{CFE})$ terpolymer (100:0), its blends with different wt. \% (5-50) of $\mathrm{P}(\mathrm{VDF}-\mathrm{TrFE})$ copolymer, and in the pure copolymer (0:100). Due to clarity the y-scale of the copolymer sample, which shows a ferroelectric behavior, is different to those of other samples, which exhibit either relaxor behavior or relaxor-ferroelectric coexistence. 


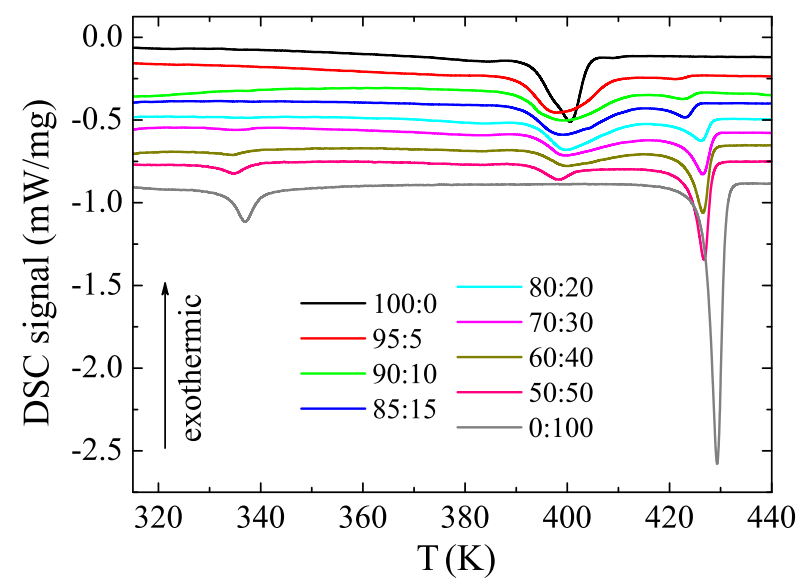

Figure 2: DSC traces of the pure $\mathrm{P}(\mathrm{VDF}-\mathrm{TrFE}-\mathrm{CFE})$ terpolymer (100:0), its blends with the $\mathrm{P}(\mathrm{VDF}-\mathrm{TrFE})$ copolymer, and in the pure copolymer $(0: 100)$. 